\title{
Many pathways to apoptosis: E2F1 regulates splicing of apoptotic genes
}

\author{
K Korotayev ${ }^{1}$ and D Ginsberg ${ }^{*, 1}$ \\ Cell Death and Differentiation (2008) 15, 1813-1814; doi:10.1038/cdd.2008.155
}

\begin{abstract}
E2F transcription factors are best known for their ability to determine the timely expression of many genes required for entry into and progression through the $S$ phase of the cell cycle. However, it is now clear that E2Fs function in a wide range of biological processes, including DNA replication, mitosis, the mitotic checkpoint, DNA damage checkpoints, DNA repair, differentiation and development. ${ }^{1}$
\end{abstract}

In addition, at least one member of the E2F family, E2F1, can induce apoptosis through a number of p53-dependent and -independent mechanisms. ${ }^{2}$ E2F1-induced apoptosis is attributed mainly to E2F-mediated upregulation of various proapoptotic genes. The growing list of death-inducing E2F1regulated genes includes genes encoding Apaf1, caspases, BH3-only proteins, the p53 family member p73 and p53 proapoptotic cofactors such as ASPP1 and ASPP2. ${ }^{2}$ Certain studies show that transactivation-defective E2F1 mutants are capable of inducing apoptosis efficiently, indicating that, at least in some settings, E2F1 employs transactivationindependent mechanisms to promote apoptosis. Indeed, as well as transactivating gene expression, E2F1 is capable of derepressing and repressing gene expression. For example, E2F1 can sensitize cells to apoptosis by inhibiting survival signals, in particular those mediated by the transcription factor $\mathrm{NF}-\kappa \mathrm{B}$ or by $\mathrm{Bcl}-2$ and its family member $\mathrm{Mcl}-1$, the latter encoded by a gene transcriptionally repressed by E2F1. Thus, through a combination of activation, derepression and repression of transcription, E2F1 regulates gene expression and directs apoptosis. Furthermore, E2F1 has been proposed to possess transcription-independent activities that promote apoptosis. $^{3}$

A new paper in Cell Death and Differentiation extends our understanding of how E2F1 shapes gene expression to affect apoptosis. ${ }^{4}$ Eymin and colleagues ${ }^{4}$ show that E2F1 controls alternative splicing of apoptotic genes through direct transcriptional upregulation of the splicing factor SC35 (also named SFRS2), a member of the serine/arginine-rich (SR) family of splicing factors. Members of the SR family play vital roles in both constitutive splicing and regulated alternative splicing. Apoptosis is one of the cellular processes in which alternative splicing plays a pivotal regulatory role: a large number of apoptotic factors are regulated by alternative splicing that results in the production of discrete protein isoforms with often antagonistic functions, namely pro- and antiapoptotic. ${ }^{5}$ Indeed, a number of splicing factors have been shown earlier to influence apoptosis. For example, depletion of SF2/ASF induces apoptosis and, conversely, its ectopic expression protects from apoptosis. ${ }^{6,7}$ Also, ectopic expression of SC35 was shown to affect alternative splicing of caspase 2 and to induce apoptosis. ${ }^{8}$ Moreover, recent studies have suggested that splicing can sometimes be coupled to transcription. In particular, some transcription factors bind components of the spliceosome or exhibit dual functions in splicing and transcription. ${ }^{9}$ However, the work of Eymin and colleagues identifies a different effect of a transcription factor on splicing, namely that E2F1 regulates expression of the splicing factor SC35, which in turn determines the ratio of proapoptotic to antiapoptotic splice variants.

The capacity of E2F1 to regulate SC35 expression is demonstrated by the positive and negative effects of ectopically elevated and reduced E2F1 levels, respectively, on SC35 expression. The direct nature of this SC35 regulation is supported by data showing E2F1 binds and activates the SC35 promoter. Importantly, knockdown of SC35 inhibits E2F1-induced apoptosis, indicating that SC35 is a pivotal mediator of E2F1-induced apoptosis. ${ }^{4}$ E2F1 was reported earlier to downregulate one isoform of the cellular FLICEinhibitory protein, c-flip short, and thereby to activate caspase 8 at the death inducing signaling complex (DISC) and sensitize cells to death ligands such as FasL and TRAIL. ${ }^{10}$ The relationship between E2F1 and SC35 uncovered by Eymin and colleagues now provides the mechanism underlying this earlier observation. Indeed, knocking down SC35 inhibits the ability of E2F1 to alter the FLIP $_{\text {long }} / F L I P_{\text {short }}$ ratio in favor of the longer isoform and concomitantly reduces the ability of E2F1 to sensitize cells to death ligands. Notably, the SC35mediated ability of E2F1 to bias alternative splicing of apoptotic genes in favor of the pro-apoptotic splice variant is not limited to c-FLIP and occurs also for Bcl-x and caspases 8 and 9. Furthermore, DNA damage induces an E2F1-mediated increase in the protein levels of SC35 and, correspondingly, an increase in the pro-apoptotic splice variants of the aforementioned genes. Knockdown of either E2F1 or SC35 abolishes these changes in splicing and inhibits DNA damage-induced apoptosis. Thus, the study of Merdzhanova

\footnotetext{
${ }^{1}$ The Mina and Everard Goodman Faculty of Life Science, Bar Ilan University, Ramat Gan, Israel

${ }^{*}$ Corresponding author: D Ginsberg, The Mina and Everard Goodman Faculty of Life Science, Bar llan University, Ramat Gan 52900, Israel. Tel: + 97235318804 ; Fax: + 9723 7384058; E-mail: ginsbed@mail.biu.ac.il
} 
et al. reveals that E2F1-controlled pre-mRNA splicing events affect DNA damage-induced apoptosis.

Future studies may tell whether the E2F1-SC35 link represents a unique case or just the tip of the iceberg with respect to cross-talk between E2F and splicing. Such crosstalk may include (1) E2F-mediated transcriptional regulation of additional splicing factors; (2) a broader effect of E2F on splicing of apoptosis-related as well as proliferation-related genes; (3) physical interactions between E2F and spliceosome components and (4) effects of splicing factors on the splicing and activities of E2Fs themselves. This latter possibility is supported by studies showing that one of the dimerization partners of E2Fs, DP2, undergoes alternative splicing that affects its subcellular localization, ${ }^{11}$ and by the observation that E2F6 exhibits alternatively spliced forms. ${ }^{12}$ Moreover, studies in Drosophila suggest that splicing critically affects the activity of dE2F2, which functions mainly in transcriptional repression. $\mathrm{dE} 2 \mathrm{~F} 2$ protein levels are severely reduced in cells deficient for the splicing factor SR protein B52 (ortholog of SFRS6), most probably due to defective splicing of the de2f2 pre-mRNA. ${ }^{13}$

In accordance with the data of Merdzhanova et al., several high-throughput assays have suggested that SC35 is an E2F-regulated gene. Furthermore, in support of broader E2Fsplicing cross-talk, high-throughput assays suggest that additional splicing factors are regulated by E2F, including SFRS10, SFRS9 (SRp30c), SFRS17a (XE7), SRP20 (SRFS3), SFRS7 (SR protein 9G8) and SFPQ (PSF) ${ }^{14-16}$ However, these observations have not been investigated further and possible roles for these splicing factors in E2Fmediated biological processes, such as proliferation or apoptosis, remain to be determined. Expression of the splicing factor SRP20 (SRFS3) was suggested earlier to be E2F-regulated; ${ }^{17}$ however, Merdzhanova et al. demonstrate that expression of SRP20 is not E2F-regulated. ${ }^{4}$ These seemingly contradicting data may represent tissue- and/or species-specific differences in the SRP20 regulation.
Of note, caspases 8 and 9 are transcriptional targets of E2F1, and Eymin and colleagues now show that their splicing is regulated by E2F1. Thus, E2F1 regulates two distinct steps in the generation of these pro-apoptotic proteins. Several additional E2F-regulated apoptotic genes, such as Bim, Apaf1 and Smac/Diablo, undergo alternative splicing and it is tempting to speculate that their splicing may also be E2F1regulated.

The indirect effect of E2F on splicing, reported by Eymin and colleagues, exemplifies the capacity of E2F to affect posttranscriptional processes. Recent reports have described the ability of E2F to regulate microRNA expression, thereby affecting RNA stability and translation ${ }^{18-20}$ Taken together, these studies suggest that an important, and hitherto unrecognized, feature of $\mathrm{E} 2 \mathrm{~F}$ function is the regulation of post-transcriptional processes. E2F1 is deregulated and hyperactive in most, if not all, human tumors and levels of some splicing factors, including SC35, are elevated in some tumors. Future studies should evaluate the role of E2F-dependent post-transcriptional events in tumorigenesis.

1. DeGregori J, Johnson DG. Curr Mol Med 2006; 6: 739-748.

2. laquinta PJ, Lees JA. Curr Opin Cell Biol 2007; 19: 649-657.

3. Bell LA, O'Prey J, Ryan KM. Oncogene 2006; 25: 5656-5663.

4. Merdzhanova G et al. Cell Death Differ 2008; 15: 1815-1823.

5. Schwerk C, Schulze-Osthoff K. Mol Cell 2005; 19: 1-13.

6. Li X, Wang J, Manley JL. Genes Dev 2005; 19: 2705-2714.

7. Karni R et al. Nat Struct Mol Biol 2007; 14: 185-193.

8. Jiang ZH et al. Proc Natl Acad Sci USA 1998; 95: 9155-9160.

9. Kornblihtt AR. Adv Exp Med Biol 2007; 623: 175-189.

10. Salon C et al. Cell Death Differ 2006; 13: 260-272.

11. de la Luna $S$ et al. J Cell Sci 1996; 109: 2443-2452.

12. Dahme T et al. Eur J Biochem 2002; 269: 5030-5036.

13. Rasheva VI et al. Mol Cell Biol 2006; 26: 3468-3477.

14. Muller $\mathrm{H}$ et al. Genes Dev 2001; 15: 267-285

15. Ren B et al. Genes Dev 2002; 16: 245-256.

16. Young AP, Nagarajan R, Longmore GD. Oncogene 2003; 22: 7209-7217.

17. Jumaa H, Guenet JL, Nielsen PJ. Mol Cell Biol 1997; 17: 3116-3124.

18. Petrocca $\mathrm{F}$ et al. Cancer Cell 2008; 13: 272-286.

19. Sylvestre $Y$ et al. J Biol Chem 2007; 282: 2135-2143.

20. Woods K, Thomson JM, Hammond SM. J Biol Chem 2007; 282: 2130-2134. 